\title{
Laser-generated shocks and bubbles as laboratory-scale models of underwater explosions
}

\author{
Theodore G. Jones ${ }^{\mathrm{a}}$, Jacob Grun ${ }^{\mathrm{a}}$, L. Dale Bibee ${ }^{\mathrm{b}}$, Charles Manka ${ }^{\mathrm{c}}$, Alexandra Landsberg ${ }^{\mathrm{d}}$ and \\ Daniel Tam ${ }^{\mathrm{d}}$ \\ ${ }^{a}$ Naval Research Laboratory Plasma Physics Division Washington, DC 20375, USA \\ ${ }^{\mathrm{b}}$ Naval Research Laboratory Naval Oceanographic Office Stennis Space Center, MS 39522, USA \\ ${ }^{\mathrm{c}}$ Research Support Instruments, Inc. 4325-B Forbes Blvd. Lanham, MD 20706, USA \\ ${ }^{\mathrm{d}}$ Naval Surface Warfare Center, Indian Head Division Indian Head, MD 20640, USA
}

Received 19 January 2002

Revised 3 September 2002

\begin{abstract}
Underwater shocks and bubbles were generated using a high energy pulsed laser system. The advantages of this experimental approach are: (1) precisely controlled and measured experimental conditions; (2) improved diagnostics, including extensive imaging capabilities; (3) unique experiments, including a simultaneously detonated line charge; and (4) the ability to provide validation quality data for hydrodynamic simulation codes. Bubble sensitivity to variation of several experimental parameters was examined. Numerical simulations were performed corresponding to the experimental shots, showing that empirical bubble theory, experimental bubble data, and simulations were all in good agreement.
\end{abstract}

\section{Introduction}

Laboratory-scale laser-generated shocks and bubbles have previously not been applied to modeling of underwater explosion shock and bubble dynamics; however, this approach is an excellent source of data for validation of simulation tools. The present approach is unique in providing both shock and bubble dynamics data in free-field conditions. The Laboratory for Underwater Hydrodynamics (LUH) at the Naval Research Laboratory utilizes a $1 \mathrm{~kJ}, 5$-ns duration pulsed laser, which is focused to a small spot inside a pressurized water tank to generate kbar, cm-scale spherical shocks. The LUH is characterized by: its precisely controlled and measured experimental conditions; a complete set of diagnostics, including extensive imaging capabilities; its ability to be rapidly reconfigured for different tests; and by its low cost/experiment and high repetition rate, compared with explosive tests. These laboratory-scale tests are designed to provide validation-quality data to verify numerical simulation codes such as DYSMAS/Gemini, one of the main predictive codes for the Navy. Initial feasibility experiments with laser-generated shocks and bubbles have been documented [1]. An important goal of the experimental work presented here is to demonstrate the validity of laser-generated shocks and bubbles as scaled models of underwater explosions by comparison with detailed hydrocode simulation.

Although previous laser-generated shocks have been used to study underwater cavitation hydrodynamics [2], those experiments were typically conducted with laser energies on the order of a few $\mathrm{mJ}$ of energy, and the purpose was the study of shock hydrodynamics associated with ocular laser surgery. Spark-generated shocks and bubbles have also been demonstrated for the scaled laboratory study of underwater explosion hydrodynamics [3]. However, spark-generated bubble studies require the presence of electrodes which may interfere with bubble dynamics, whereas laser-generated bubbles do not require hardware near the shock source for 
certain configurations such as shocks generated at a sediment-water boundary. Finally, previous laser- and spark-generated bubble experiments did not include studies of shocks and pressure measurements.

LUH experiments include the study of shock propagation through partially saturated underwater sand bottoms and the investigation of the interaction of reentrant bubble jets with the sand bottom surface, including cratering phenomena. This work supports the development of a predictive capability of Navy hydrocodes for mine countermeasures systems. By adequately controlling and measuring the sand bottom conditions, experiments can be designed to assess their effect on the performance of explosives on the bottom. In addition, LUH experimnets include the detailed study of the interaction of bubbles with rigid plates, in support of the Undersea Weapons program.

Experiments described here were diagnosed with high-speed multi-frame photographic imaging, both standard and schlieren shadowgraphic single-frame imaging using CCD cameras and pulsed laser backlighting, fiber-optic and electric pressure gauges. For purposes of validating numerical simulation codes, these diagnostics are used to produce a complete and precise set of data of the relevant physical response phenomena. The goals of the numerical simulations were, first, to understand how the initial conditions for the simulations correspond to the experimental initial conditions, and then how the initial conditions affect shock pressure and bubble dynamics. Data sets include pressure time histories as well as time-resolved visual recording of the bubble evolution and interaction with the sand bottom. In this paper, we will show that for free-field shock and bubble dynamics, empirical bubble theory, the LUH experimental bubble data, and the NSWC-IHD simulations are all in good agreement.

\section{Experimental setup}

Underwater shocks at the LUH are generated by shining a pulsed laser onto an solid, opaque target in a water chamber. The LUH utilizes the Pharos laser system, a high-energy high-power pulsed glass laser, for this purpose. This system is a Nd:glass laser, operating at a wavelength of $1054 \mathrm{~nm}$, and producing pulses with up to $500 \mathrm{~J}$ of energy and $5 \mathrm{~ns}$ duration. These pulse lengths and energies translate into a laser power of $100 \mathrm{GW}$. The laser pulse duration is much shorter than shock propagation or hydrodynamic timescales, and ensures that energy is deposited instantaneously

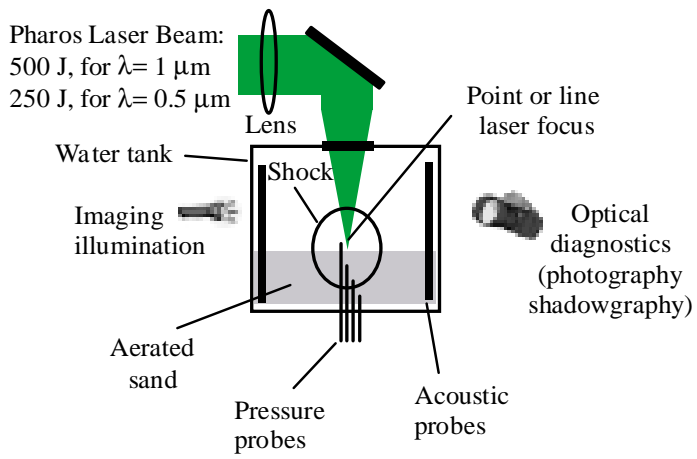

Fig. 1. A schematic of the laser shock generation.

at the laser target, from a hydrodynamics standpoint. The Pharos laser system can be fired at full energy at a repetition rate of up to once every 50 minutes, an improved repetition rate, compared to field experiments using explosives. This repetition rate is determined by the cooling time necessary for the glass laser amplifiers.

Infrared light at $1054 \mathrm{~nm}$ wavelength has a very short absorption length in water of only a few $\mathrm{cm}$. Therefore, it is necessary to convert the light to green light with $527 \mathrm{~nm}$ wavelength. At $527 \mathrm{~nm}$, the absorption length of light in water is approximately $50 \mathrm{~m}$, much longer than that for infrared light. Wavelength conversion is done by passing the beam through a Type II $\mathrm{KD}^{*} \mathrm{P}$ frequency doubling crystal, a nonlinear optical device which converts up to $55 \%$ of the energy into $527 \mathrm{~nm}$ light. Wavelength-dependent mirrors then selectively reflect the green light from this mix of infrared and green light into the experimental chamber, allowing most of the remaining infrared light to pass through into beam dumps. This technique ensures that the laser pulse entering the chamber is predominantly green light. A series of at least two wavelength-selective mirrors were used in these experiments.

Before entering the experimental chamber, the laser beam passes through focusing optics. A schematic of the experimental setup is shown in Fig. 1. For point shock generation, a spherical lens system with effective focal length of $2 \mathrm{~m}$ was used. This produces a pointshaped laser focus. Depending on where the target was placed along the beampath, the area of laser energy deposition was varied from a circle with diameter of approximately $100 \mu \mathrm{m}$ up to one of several $\mathrm{mm}$. The beam entered the chamber through a side window for free-field point shock experiments, and through a window on top of the chamber for line focus experiments.

The experimental chamber consists of a cylindrical stainless steel tank, with several window ports for laser and diagnostic access, and a lower section which 


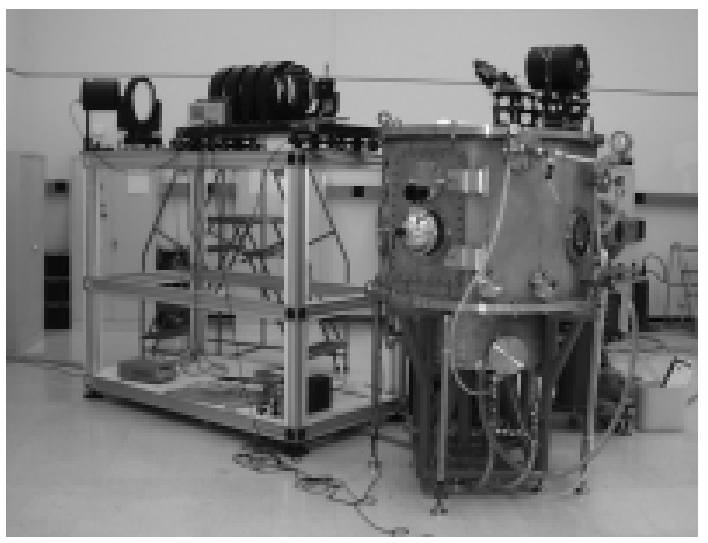

Fig. 2. A photograph shows laser optics and the setup is shown. LUH experimental chamber (right).

can accommodate sand samples. A photograph of the chamber is shown in Fig. 2. The chamber is vacuumtight and can be pressurized to 2 atmospheres (bar), or pumped down to 70 Torr of vacuum $(0.1 \mathrm{bar})$. Depth effects can be studied at pressures up to 2 bar, and bubble dynamics imaging can be enhanced by enlarged bubble formation in a partial vacuum.

The water in the chamber can be circulated through external filters and deionizing cartridges to allow clear imaging and use of electrical pressure gauges in the water. The water conditioning system can also generate de-aired, gas saturated, or super-saturated water, depending on required sediment air content.

The laboratory tank at NRL was carefully designed to carry out sand shock propagation and bubble-plate interaction experiments. A removable sand tray was designed with a porous bottom that allows water to flow through the sand under small pressure gradients. The tray and sand samples are disc-shaped, with a diameter of $75 \mathrm{~cm}$ and a depth of $20 \mathrm{~cm}$. The height of the surface of the sand sample is adjustable for precise alignment with imaging diagnostics. By controlling the tank pressure and gas saturation level of the water, we can produce uniform and stratified distributions of gas within the sand spanning those levels observed in natural environments. For bubble-plate interaction experiments, a horizontal metal plate, $30 \mathrm{~cm}$ diameter by $6.4 \mathrm{~mm}$ thickness can be placed in the chamber, at an adjustable height.

\section{Diagnostics}

\subsection{Pressure gauges}

Two types of pressure gauges were used in LUH experiments: (1) carbon piezoresistive stress gauges; and (2) optical fiber-coupled MEMS etalon pressure sensors. The carbon pressure gauges used in these experiments were piezoresistive. Low-noise folded-lead gauges, model FC300-50-EK from Dynasen, Inc., Goleta, CA were used. These gauges have a pressure-sensing carbon element of dimension $1.3 \mathrm{~mm} \times 2.6 \mathrm{~mm} \times 13 \mu \mathrm{m}$ thick. For changes in resistance of less than $20 \%$ (corresponding to pressures of 6 kbar or less), change in resistance is linearly dependent on pressure. The resistive response of these gauges is calibrated with $\mu$ s-timescale projectile-impulse tests in the range of 20 bar to 50 kilobar.

Gauge resistance was measured using an impedance bridge (in Wheatstone quarter bridge configuration), with a gated power supply driving $120 \mathrm{~mA}$ through the test gauge. One leg of the impedance bridge consisted of a potentiometer for balancing the zero-pressure voltage. Resistance was determined from bridge voltage using the nonlinear analytic formula for the response of the impedance bridge. Several EMP noise reduction techniques were employed, including the use of: rigid coaxial signal cables, high-inductance ferrite beads on the coaxial cables, spatial isolation from the plasma shock source which provides a time delay for shock arrival sufficient for EMP noise to decay, and special signal clipping circuitry which minimized amplifier saturation during the brief initial EMP pulse.

A second type of pressure gauge, based on a fiber optic-coupled Fabry-Perot interferometer, was developed for use in LUH experiments. This optical gauge produces measurements with better time resolution and significantly reduced electrical noise, and has replaced carbon gauges in LUH experiments. The pressuresensitive element within the device consists of an optical cavity with a partially reflective coating on the inside, one side of which is a deformable membrane. Reflectivity back into the fiber is a function of the optical cavity spacing. The membrane deformation, cavity spacing, and therefore reflectivity are determined by the external pressure. These gauges are illuminated by a laser diode, via the optical fiber. The reflected light is then measured with a ns-response photodiode to determine pressure. These gauges are sensitive to pressures from 0 to 400 bar. The optical cavity element is $213 \mu \mathrm{m}$ in diameter, with a resonant frequency on the order of $10 \mathrm{MHz}$. These optical cavity gauges, model POI-400/SP, were manufactured by Photonetics, Inc., Peabody, MA.

\subsection{Imaging}

Movies of bubble collapse dynamics were made with a Cordin model 377 high-speed drum camera. This 
camera can record 500 frames at any speed between 1,000 and 200,000 frames per second. A counterrotating film drum and image relay mirror operate in a vacuum to reduce heating and power requirements. Two distinct types of shuttering are used in the Cordin. One is provided by the capping shutter, which controls the total writing time. The second type of shuttering is provided by the rotating mirror in conjunction with rectangular stops and the film fixed to the counterrotating drum. The exposure time thus depends upon the camera rotational speed.

The bubbles are backlit with a high-intensity MeggaFlash magnesium flashbulb, which has a light output of 5 million lumens in $15 \mathrm{~ms}$. This light passes through a glass diffuser located $10-20 \mathrm{~cm}$ from the laser target, on the side opposite the drum camera. The flashbulb, drum camera shutter, and Pharos laser pulse are synchronized and timed by a set of digital delay generators.

Images of the shock wave in the water were taken using schlieren imaging [4]. Schlieren imaging (also known as dark-field shadowgraphy) has been used extensively in Pharos laser-generated shock experiments. A system producing one schlieren image per shot was used in these experiments. A pulsed probe laser with arbitrary timing and $8 \mathrm{~ns}$ pulse duration is used to strobe the region containing the shock and freeze the shock motion. Collimated light from this probe beam passes through the region of interest in the target chamber and exits through another window to be collected by imaging optics. In the target chamber, shock propagation through transparent media creates density gradients. These gradients cause deviations in the directions of the rays of the collimated probe beam. A lens brings the undeviated rays in the probe beams to a focus after passing through the target. A small mask is then placed at the focal point to block all undeviated rays. This allows only information-bearing (deviated) rays to pass to the image-recording CCD camera. The resulting image shows a bright outline of the shock front, with a dark background.

\section{Experimental results}

\subsection{Laser shock generation}

Our experiments demonstrated successful focusing in water at $\mathrm{f} / \#$ in the range 1.6-4.7 (where $\mathrm{f} / \#$ is defined as the laser focal length divided by the beam diameter at the focusing lens), and for energies up to $200 \mathrm{~J}$.
No pinching or other beam instability was observed in visible images before the beam reached focus.

Free-field laser shock generation experiments without a laser target yielded a shock source that was elliptical in shape and a few centimeters in length, corresponding to the volume of water containing the beam focal waist and high laser intensities. In free-field and bubble-plate interaction experiments, an opaque foil target was placed in the laser beam near the focus in order to better localize the energy deposition and shock source. The foil was typically a square of plastic (either Kapton or Upilex) $8 \mathrm{~mm} \times 8 \mathrm{~mm}$ and between 25 and $75 \mu \mathrm{m}$ thick. Plastic was chosen as a target material in order to roughly match the shock impedance and density of water. The laser energy was absorbed at the surface of the foil target, creating better-localized shock sources. Experiments without a laser target also revealed that near the laser focus, the light intensity was sufficient to ionize the water. After sufficient ionization of the water, light will be reflected by the conductive plasma, preventing further absorption of laser energy at the shock source. To prevent this, the laser target was typically placed in the laser beam path ahead of the focus, so that the laser formed a circular spot between 5 and $8 \mathrm{~mm}$ in diameter on the target.

At the highest laser intensities $\left(I>1010 \mathrm{~W} / \mathrm{cm}^{2}\right)$, some beam energy was deposited in the focal volume in front of the beam focus, as evidenced by shocks generated at the boundaries of this conical volume. These were interpreted as being caused by rapid heating and expansion of the water in the focal volume during the 5 ns laser pulse. In schlieren images, the shock generated by the focal cone can be seen to propagate at the sound speed in water from the earliest observation times $(t<1 \mu \mathrm{s})$. These slow early shock speeds indicate much lower pressures (vavg within $10 \%$ of sound speed within in the first $3 \mathrm{~mm}$ of propagation, corresponding to $\bar{p}<1 \mathrm{kbar}$ ) compared to the shock generated by the laser plasma at the target. In addition, this shock does not form a large bubble as does the laser plasma at the target. Finally, bubble dynamics were not observed to be affected by these shocks.

All laser energies quoted in this paper are the $527 \mathrm{~nm}$ pulse energy at the window, where the beam enters the experimental chamber. The bubble sizes observed in LUH experiments with laser energies from $100-200$ Joules have typical bubble sizes of approximately $4 \mathrm{~cm}$ diameter, corresponding to a bubble mechanical energy on the order of 1 Joule at atmospheric pressure. Therefore, most of the laser energy is scattered or absorbed in the water before reaching the laser target. Strong 

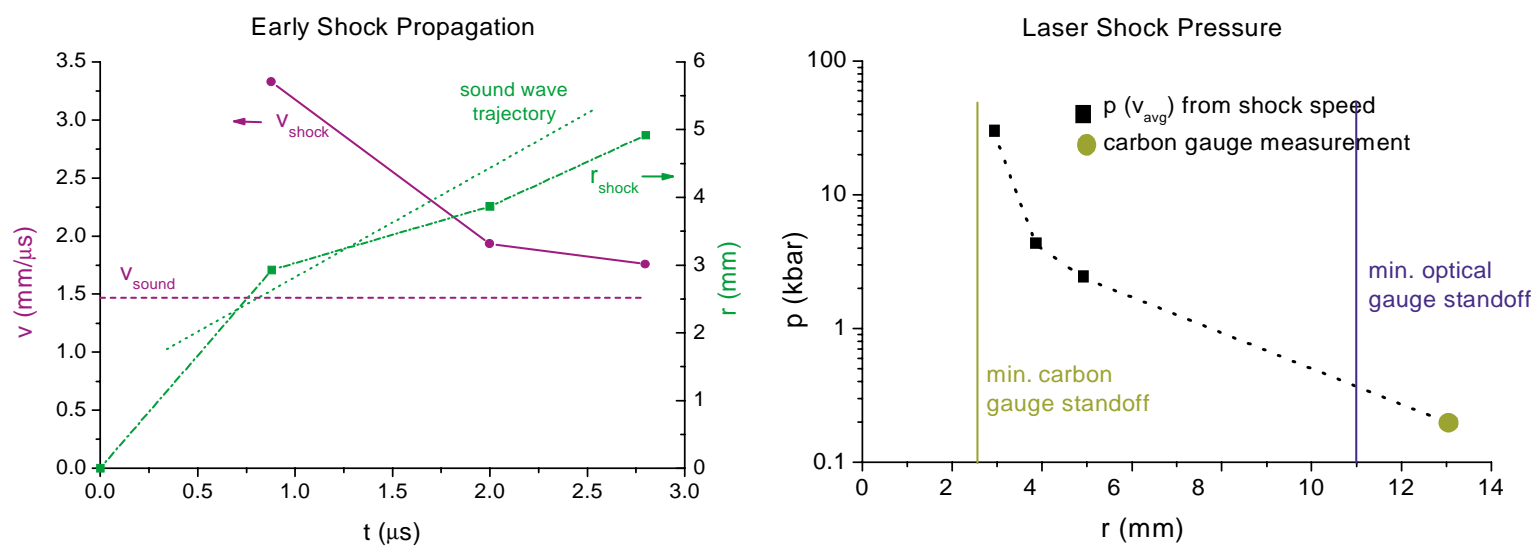

Fig. 3. The left-hand graph shows shock radius as a function of time (dot-dashed line), and average velocity to shock-front location (solid line), inferred from schlieren shock imaging data at early times $(t<3 \mu \mathrm{s})$. Each shock front image was taken during a different experimental shot with the same experimental parameters. The right-hand graph shows the "average pressure", $p($ żg $)$ at each imaged shock front location, as well as the closest carbon gauge pressure measurement. The minimum standoff distance for LUH pressure gauges (corresponding to maximum pressures) are indicated.

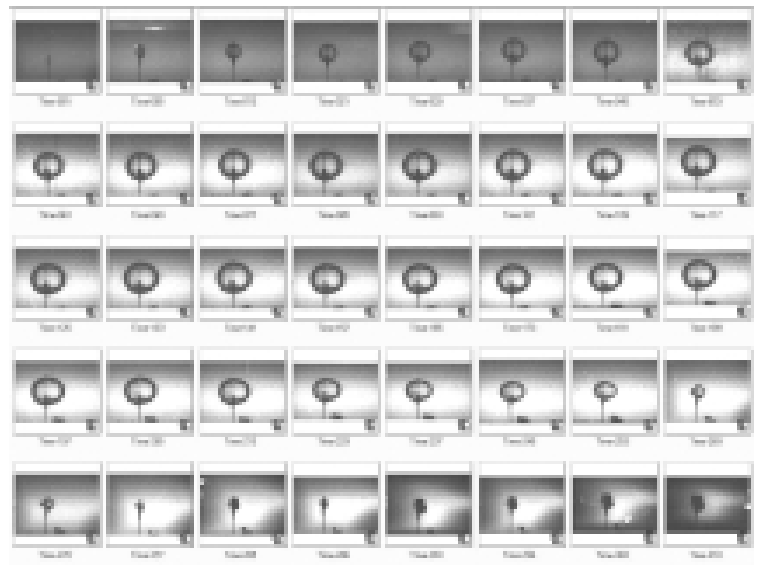

Fig. 4. A subset of images from a typical free-field.

candidates for nonlinear optical scattering mechanisms include molecular Brillouin and molecular Raman scattering in water, as well as specular reflection of laser light from any ionized volumes of water near the surface of the laser target. An investigation of these scattering phenomena in LUH experiments is ongoing.

\subsection{Shock dynamics}

Laser-generated shocks from solid targets were imaged over a range of laser energies up to $150 \mathrm{~J}$ using schlieren imaging. For a given laser energy and focal spot size, these shocks had reproducible propagation speeds and shapes. Three shots were taken with the same laser energy and target. Shock propagation distances at $15 \mu \mathrm{s}$ (which determine the average shock speeds from $t=0$ ) were within $10 \%$ of each other for a spread in laser pulse energy of approximately $10 \%$. At small radii, the shocks formed a pancake-shaped ellipsoid in the plane of the foil and centered on the beam axis. The shocks became spherical at radii greater than the approximate dimension of the foil (approximately $8 \mathrm{~mm}$ for the $2 \mathrm{~mm} \times 7 \mathrm{~mm}$ Upilex targets).

The shock pressure at small radii $(r<5 \mathrm{~mm})$ and early times $(t<3 \mu \mathrm{s})$ was inferred from shock propagation distances measured using schlieren imaging. This was the only available technique for determining pressure at the innermost radii, as existing gauges are not suited to the small spherical shocks and high pressures this close to the shock source. At $t=880 \mathrm{~ns}$ after the Pharos beam pulse, the shock radius was $2.9 \mathrm{~mm}$, yielding an average shock expansion velocity, from time $t=0$, of $v_{\text {avg }} r / t=3.3 \mathrm{~mm} / \mu \mathrm{s}$. Shock pressure can be related to shock speed using the empirical formula [5]:

$$
p=\left(\frac{1}{2.1}\right) \rho_{o} v\left(v-v_{s}\right)
$$

where $\rho_{o}$ is the unperturbed water density, $v_{s}$ is the speed of sound in water, and $v$ is the shock speed. Substituting $\operatorname{vavg}(2.9 \mathrm{~mm})$ for $v$ yields a pressure $\bar{p} \equiv$ $p\left(v=v_{\text {avg }}\right)=29 \mathrm{kbar}$. This inferred shock pressure is valid at the radius (inside $3 \mathrm{~mm}$ ) where $v=v_{\text {avg }}$. The initial pressure at the target is higher. At $5 \mathrm{~mm}$, $v_{\text {avg }}$ was within $20 \%$ of the sound speed. At distances greater than $10 \mathrm{~mm}$ shock propagation speed was within measurement error of sound speed. Figure 3 shows a plot of shock radius and $v_{\text {avg }}$ as a function of time, as well as the pressure, determined from Eq (1). 


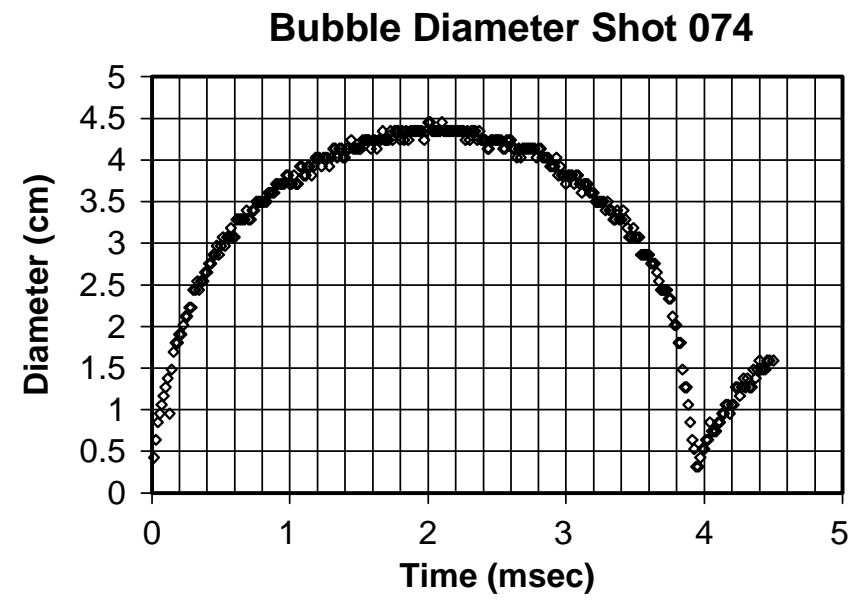

Fig. 5. Bubble diameter as a function of time is bubble evolution is shown. shown for the free-field experiment shown in Fig. 5.

The shock pressure was measured directly with a carbon gauge at a $13 \mathrm{~mm}$ distance from the laser plasma to be approximately 200 bar and is plotted in Fig. 3, at right. The same pressure was measured with an optical gauge at a similar radius on a shot with similar laser energy and identical experimental setup. The duration of the shock front at this distance was on the order of $1 \mu \mathrm{s}$.

\subsection{Bubble dynamics}

At late times $(t>100 \mu \mathrm{s})$, the initial superheated volume created by the laser was seen to expand into a spherical bubble. Over 50 shots were taken in free-field configuration with a wide range of laser energies, focal spot sizes, and with different laser target materials. Shock and bubble dynamics from these experiments were measured with a variety of pressure and imaging diagnostics, including the high-speed drum camera. A typical free-field bubble image sequence is shown in Fig. 4, with the corresponding plot of bubble diameter evolution shown in Fig. 5. For the typical laser energies of approximately $150 \mathrm{~J}$, the bubble expansion time was on the order of $1 \mathrm{~ms}$ and the maximum radius was approximately $2 \mathrm{~cm}$. Bubble radius was observed to increase with laser energy, as shown in Table 2. Experimental bubble data for two different laser energies is shown in Fig. 10. The bubble was observed to collapse again to a minimum radius at approximately $3 \mathrm{~ms}$. The beginning of the second bubble oscillation period was also recorded, as seen in Figs 4, 5 and 10. In our free-field experiments, bubbles were measured to have spherical symmetry to within $5 \%$ after expansion to 1.5 times the laser target size.

\subsection{Bubble-Rigid boundary interaction}

Once free-field scaled underwater explosions were demonstrated with laser-generated shocks, preliminary bubble jetting experiments were conducted. The principal diagnostic during these experiments was the highspeed drum camera, which was used to generate sequential images of bubble-rigid plate interactions. Image sequences were taken with three different separations between the shock source and the metal plate: $1.0 \mathrm{~cm}, 2.0 \mathrm{~cm}$, and $3.5 \mathrm{~cm}$. In dimensionless hydrodynamics units, normalized to the maximum bubble radius in free-field, these correspond to $0.5,1.0$, and 1.75 , respectively. The clearest bubble jetting phenomenon was seen with a normalized separation of 1.0. Jet velocities were in excess of $100 \mathrm{~m} / \mathrm{s}$, with the jet motion across the bubble interior not resolved in these first images.

\section{Simulation and theory}

A series of numerical simulations were performed to understand how the initial conditions for the simulations correspond to the experimental initial conditions, and how the initial conditions affect shock pressure and bubble dynamics. The simulations increased in both complexity and predictive accuracy for bubble diameter evolution, gauge pressure, and expected laser energy deposition.

The simulation code, which divides the region of interest into multiple cells for calculations, must be able to accurately model multiple materials within a cell since each may contain water, air and sand. It 


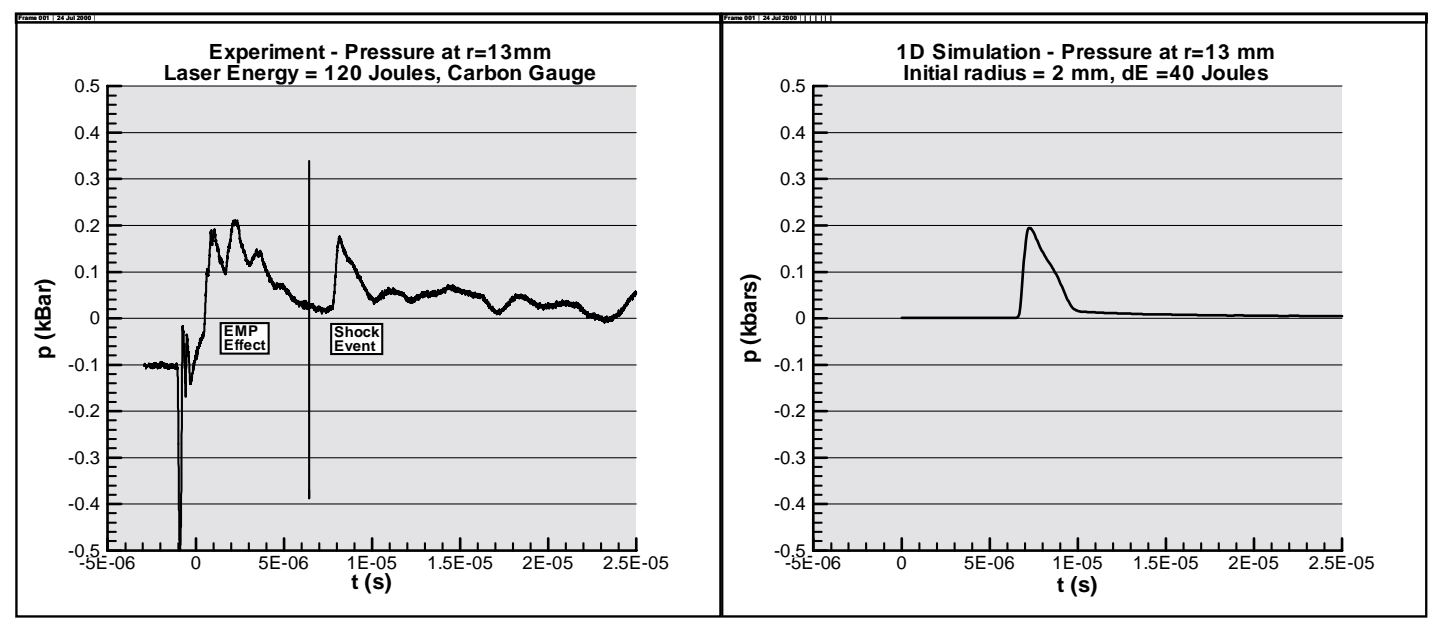

Fig. 6. A comparison of experimental shock pressure (left) and simulation shock pressure (right) at $r=13 \mathrm{~mm}$ is shown. The experimental (left) and calculated (right) pressures agree well. The initial structure in the experimental trace is electrical noise, which was eliminated by using optical pressure gauges in later experiments.

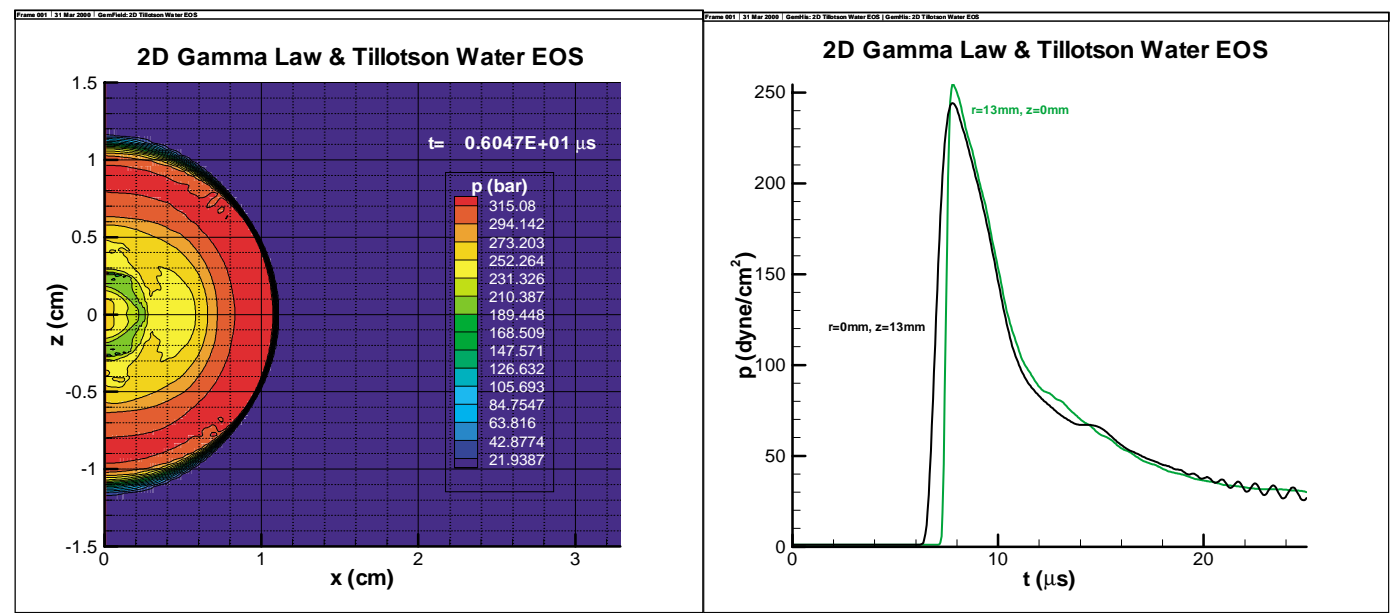

Fig. 7. Simulated pressure contours at $t=6 \mu$ s (left), and simulated pressure time history at 13mm distance for two different locations (right) are shown for a cylindrical deposition volume with 1:1 aspect ratio. The 1:1 aspect ratio can be seen to yield an isotropic shock pressure.

must also have appropriate Equations-Of-State (EOS) for modeling water and sand. A compressible code is needed to model the initial shock, but must also be able to capture the bubble dynamics. One of the goals of this work is to improve upon the existing simulation tools for the Navy. The compressible hydrocode, DYSMAS/Gemini, was chosen for the numerical simulations since it is one of the main predictive codes for the Navy.

\subsection{1-D Simulations}

Early numerical simulations of LUH data attempted to match the shock pressure time history in water gen- erated from the laser experiment [1]. In order to perform numerical simulations similar to the laser experiment, a set of initial conditions must be chosen; however, some of the initial conditions corresponding to the experiment are not known. In the simulations, an initial energy and volume define the initial conditions. A Tillotson equation of state was used to model water with the energy deposited instantaneously in the initial volume. The typical laser energy entering the experimental chamber was on the order of $100 \mathrm{~J}$. This sets an upper limit on the amount of energy to be deposited in the initial volume. As discussed in the Experimental Results section, the actual energy absorbed at the target is significantly less than $100 \mathrm{~J}$. A series of 1-D 


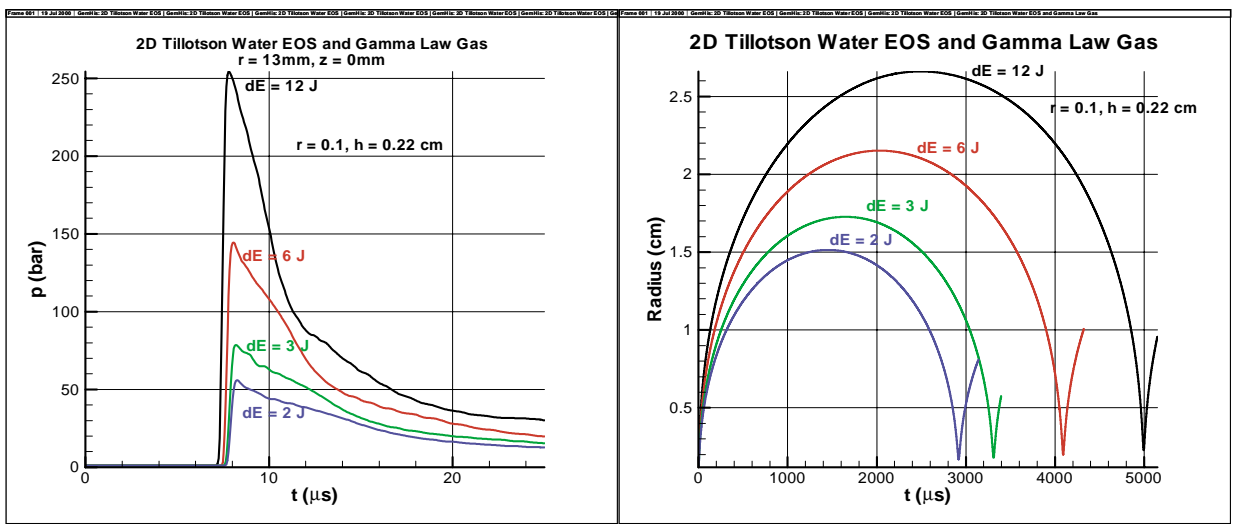

Fig. 8. A sensitivity study of shock pressure (left) and bubble dynamics (right) to initial energy is shown.

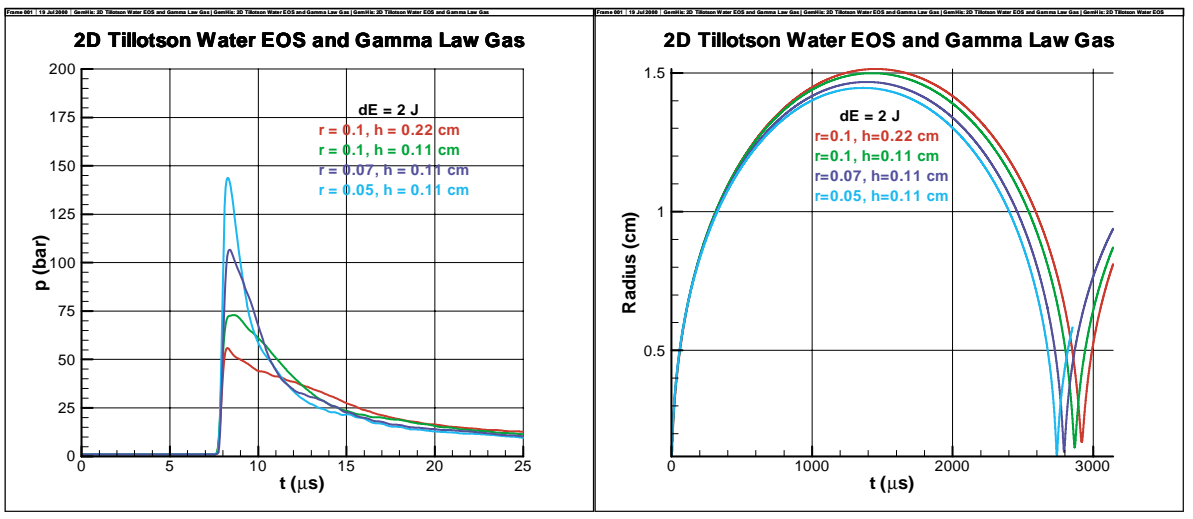

Fig. 9. A sensitivity study of shock pressure (left) and bubble dynamics (right) to initial volume is shown.

simulations varying the initial energy and radius were performed. These results show that one can match the laser experiments fairly well with this simple model. Figure 6 shows the experimental shock pressure time history from a carbon gauge at $13 \mathrm{~mm}$ from the target. A simulation with an initial radius of $2 \mathrm{~mm}$ and $40 \mathrm{~J}$ produces a similar shock profile. The large electrical noise early in the signal (before $6 \mu \mathrm{s}$ ) is typical of a carbon gauge, however, the newer optical gauges significantly reduce this noise. A problem arises with the simulations. Rather than obtaining a unique initial condition, a class of initial conditions can be made to match the experimental data based upon shock pressure data only. In order to define a unique set of initial conditions, both the shock pressure and bubble data must be used. To more precisely determine appropriate simulation initial conditions, 2D simulations were performed.

\section{2. $2 D$ simulations}

A series of 2D axisymmetric sensitivity studies were performed in which the effect on shock pressure and bubble dynamics of (1) the deposited laser energy; and (2) the energy deposition volume was examined. A Gamma Law $(\gamma=1.25)$ EOS was used to model the bubble. A simulation with 1:1 deposition volume aspect ratio (diameter:length), shown in Fig. 8, was able to reproduce the experimental data as closely as the 1D simulation.

Subsequently the effect of varying the deposited energy, dE, given a fixed initial volume was examined. The dimensions of the initial volume were chosen to be on the same order as the laser spot size, specifically $r=0.1 \mathrm{~cm}$ and $h=0.22 \mathrm{~cm}$. Figure 8 shows the simulated pressure time history at $\mathrm{r}=13 \mathrm{~mm}$, and the bubble radius as a function of time and $\mathrm{dE}$. Based on Ref. [1], the shock pressure at $r=13 \mathrm{~mm}$ was 200 bar, and the maximum bubble radius is $2 \mathrm{~cm}$ with a period of $3 \mathrm{~ms}$. For this given volume and range of initial energies, the numerical simulations can not be made to match both the shock and bubble dynamics. One sees good agreement with experiment for the bubble radius and period for $\mathrm{dE}=2 \mathrm{~J}$, however the peak pressure is 
Table 1

A comparison of bubble radius and energy ratios is shown from theory and simulation data from Fig. 8. Column 1 plus the energy deposition volume comprise the initial conditions for the simulations

\begin{tabular}{crccccc}
\hline $\mathrm{dE}(\mathrm{J})$ & $E_{\text {total }}(\mathrm{J} / \mathrm{g})$ & $R_{\max }(\mathrm{cm})$ & $\frac{E_{1}}{E_{2}}$ & $\left(\frac{R_{\max 1}}{R_{\max 2}}\right)_{\operatorname{sim}}$ & $\left(\frac{R_{\max 1}}{R_{\max 2}}\right)_{\text {theory }}$ & Error $(\%)$ \\
\hline 12 & 1758.51 & 2.661 & 2.0 & 1.236 & 1.260 & 1.9 \\
6 & 879.255 & 2.152 & 2.0 & 1.246 & 1.260 & 1.1 \\
3 & 439.627 & 1.727 & 1.5 & 1.1740 & 1.145 & 0.4 \\
2 & 293.085 & 1.514 & & & & \\
\hline
\end{tabular}

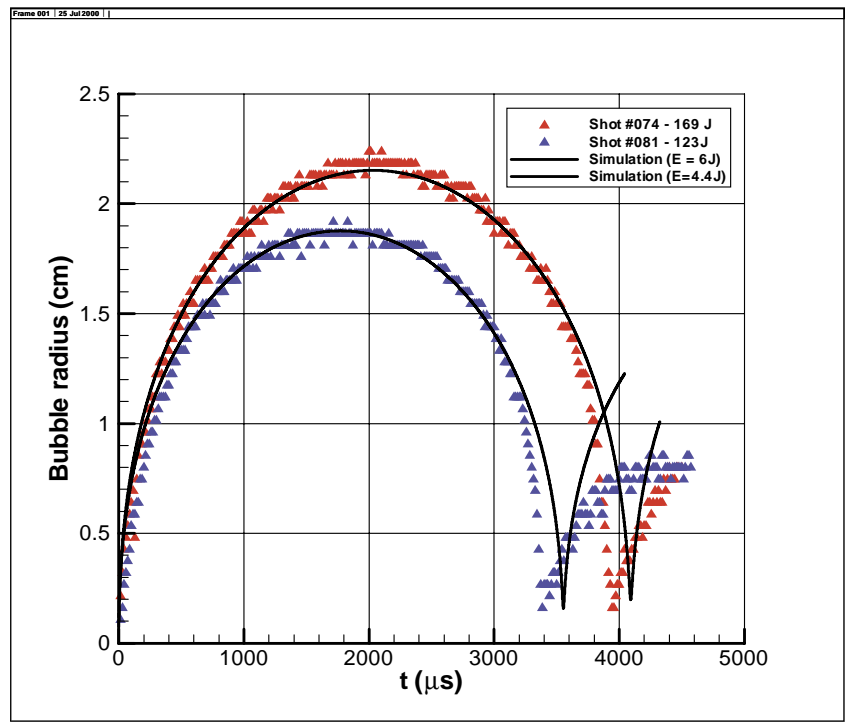

Fig. 10. A comparison of bubble dynamics from experiment (triangles) and simulation (solid lines) is shown. Bubble radii from two shots with different laser energies, but the same spot size and target material are plotted.

underestimated by almost a factor of 4 . However, the sensitivity to initial volume has not been factored into the problem.

The next series of simulations fixed the deposited laser energy, and allowed the initial volume to vary. The objective was to place the same energy in a smaller volume. The intended effect was to increase the pressure without significantly impacting the bubble radius and period. Figure 9 shows the pressure time history at $r=13 \mathrm{~mm}$ and the bubble radius as a function of time and volume for a fixed $\mathrm{dE}=2 \mathrm{~J}$. By placing the same energy in a smaller volume, the peak pressure significantly increased, as expected. The maximum volume varies by a factor of 8 , with the result that the peak pressure at $r=13 \mathrm{~mm}$ increases by a factor of approximately 2.6 but the bubble radius only changes by factor of approximately 5\%. So, a variation of the energy deposition volume and volume shape yield an accurate simulation of the bubble dynamics and shock pressure for a given laser target and spot size. More generally, these results indicate that the deposited laser energy, dE, strongly impacts the shock pressure and bubble dynamics while the initial volume strongly impacts shock pressure, but has a smaller effect on the bubble dynamics.

\subsection{Comparison between theory, simulation and experiment}

There is extensive theoretical and experimental analysis of the behavior of underwater explosion bubbles (Ref. Cole [6], etc.). Theoretical relationships exist between bubble maximum radius, bubble period, total energy, and water depth. The maximum bubble radius Rmax is related to the total deposited laser energy, E, by the relation:

$$
R_{\max } \propto E^{1 / 3} P_{0}^{-5 / 6},
$$

where $P_{0}$ is the hydrostatic pressure. The goals are to determine if (1) the simulations match theory and (2) the experiments match the simulations and theory. One can take the ratio of two (laser) energies, and use 
Table 2

Comparison of experiment and simulations at $t=1.0 \mathrm{~ms}$. $E_{\text {laser }}$ is the laser energy into the water chamber, and is assumed to be proportional to the energy coupled into the bubble at the laser target. Experiment and simulation agree to within $2 \%$

\begin{tabular}{ccccccc}
\hline$E_{\text {laser }}$ & $R_{t=1.0 \mathrm{~ms}}$ & $\left(\frac{E_{1}}{E_{2}}\right)_{\text {expt }}$ & $\left(\frac{R_{1}}{R_{2}}\right)_{\text {expt }}$ & $\left(\frac{E_{1}}{E_{2}}\right)_{\operatorname{sim}}$ & $\left(\frac{R_{1}}{R_{2}}\right)_{\operatorname{sim}}$ & Error $(\%)$ \\
\hline 205.5 & 2.155 & 1.99 & 1.181 & 2.00 & 1.177 & 0.3 \\
169.5 & 2.040 & 1.64 & 1.118 & 1.67 & 1.132 & 1.2 \\
135.3 & 1.925 & 1.31 & 1.055 & 1.33 & 1.073 & 1.7 \\
103.1 & 1.825 & & & & & \\
\hline
\end{tabular}
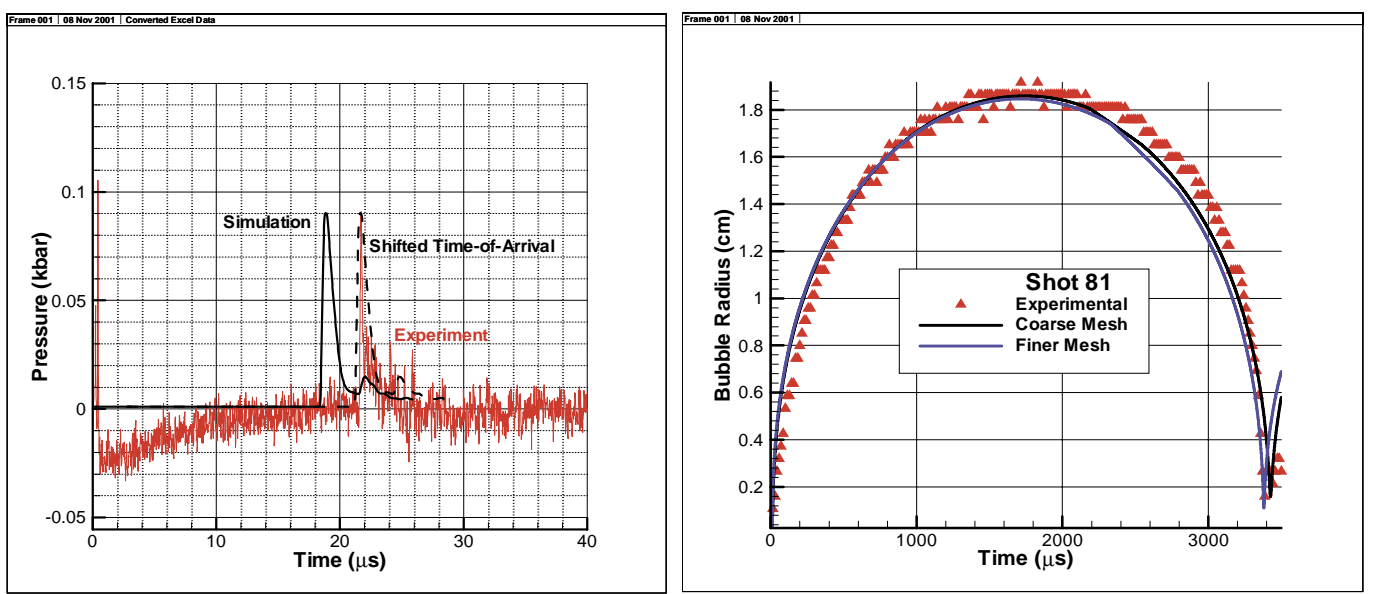

Fig. 11. A comparison of experimental results with computational results is shown. The figure on the left shows a comparison of the shock pressure time-history from simulation with that measured by an optical pressure gauge. The figure on the right shows the bubble dynamics.

Eq. (2) to obtain the ratio of maximum bubble radii:

$$
\left(\frac{R_{1}}{R_{2}}\right)_{\max } \propto\left(\frac{E_{1}}{E_{2}}\right)^{1 / 3} .
$$

This relation holds since in the experiments, the depth is constant while in the simulations, the depth is constant and only the cases with a fixed volume are considered. Table 1 shows the maximum bubble diameter and energy ratio for four energies based on the simulations shown in Fig. 8. Using these energy ratios, the ratio of theoretical maximum bubble radii can be determined. The last column shows that the error between theory and simulation is less than $2 \%$. The laser experiments provided maximum bubble radius at energy ratios of 1.66 and 1.33 , thus simulations matching these conditions were performed. As expected, simulation and theory match.

Experimental data on the bubble dynamics was obtained using the Cordin high-speed drum camera. Figure 10 shows two shots for two laser energies. The bubble dynamics from these laser experiments match closely with simulations.

Additional bubble images were taken for a range of laser energies, using a CCD camera in combination with a short pulse probe laser for backlighting at $t=$ $1.0 \mathrm{~ms}$. Comparison to simulations at $t=1.0 \mathrm{~ms}$ were done as shown in Table 2. For these cases, the error again is below $2 \%$. Thus, we have shown that the laser bubble experiments match simulations, and as discussed above, the simulations match theory.

Compressible hydrocode simulations were performed for initial conditions corresponding to free-field LUH experiments. Figure 11 shows a comparison between the experimental results and the computational results. It is generally accepted that hydrocode simulations of underwater shocks under-predict the timeof-arrival of the shock as seen in the shock pressuretime history (Fig. 11-left). The shifted time-of-arrival is purely for visualization purposes to overlay the shock profiles. More important is that the peak pressure and impulse between experiment (optical gauge) and simulation are shown to agree qualitatively. A qualitative comparison was not possible with the large noise level due to a technical problem with the optical gauge light source on these shots. The bubble radius and period are shown on the right in Fig. 11 for a coarse mesh and a fine mesh. For each mesh the solution is mesh converged, and most importantly, agrees with experi- 
ment. Thus, given a limited set of experimental pressure traces and bubble dynamics, an appropriate set of initial conditions for numerical simulations can be determined. This significantly enhances the predictive capabilities of numerical.simulations for underwater shock and bubble dynamics.

\section{Summary}

Experimental free-field shock and bubble data, compressible hydrocode simulations, and empirical bubble theory are in good agreement. Therefore, the laser-generated shocks and bubbles are valid and useful laboratory-scale models of underwater explosions. The direct impact is in providing validation-quality data for Navy hydrocode calculations of shock and bubble interactions, both in free-field configuration, and at rigid and sandy solid interfaces. The LUH data, along with hydrocode simulations, can be an invaluable tool for helping understand several Navy hydrodynamics problems, including mine countermeasures systems and advanced undersea weapons configurations. In addition, pressure sensor technology developed in the LUH, as well as improved in sediment characterization techniques and understanding of air transport and stability in sediments, are directly applicable to field experiments.

\section{Acknowledgements}

This work was supported in part by both the Undersea Weapons Program and the Surf-zone Technology Program in the Office of Naval Research. This work was also supported by ONR base funding.

\section{References}

[1] T.G. Jones, J. Grun, H.R. Burris and C.K. Manka, Feasibility Experiments for Underwater Shock and Bubble Generation with a High-power Laser, NRL Memo. Rept. 6790-99-8317, 1999.

[2] W. Lauterborn and H. Bolle, Experimental Investigations of Cavitation-bubble Collapse in the Neighborhood of a Solid Boundary, Journal of Fluid Mechanics 72(2), 1975, 391-401.

[3] G.L. Chahine, G.S. Frederick, C.J. Lambrecht, G.S. Harris and H.U. Mair, Spark-generated Bubbles as Laboratory-scale Models of Underwater Explosions and Their Use for Validation of Simulation Tools, Proceedings of 66th Shock and Vibration Symposium, Biloxi, MS 2 (1995), 265-276.

[4] J.A. Stamper, E.A. McLean, S.P. Obenschain and B.H. Ripin, Concepts and Illustrations of Optical Probing Diagnostics for Laser-produced Plasmas, Lecture presented at the NATO Advanced Study Institute on Fast Electrical and Optical Diagnostic Principles and Techniques, Lucca, Italy, 1983.

[5] Yu S. Yakovlev, Explosion Hydrodynamics (in Russian), Sudpromgis, Leningrad, 1961.

[6] R.H. Cole, Underwater Explosions, Dover Publications, 1948. 

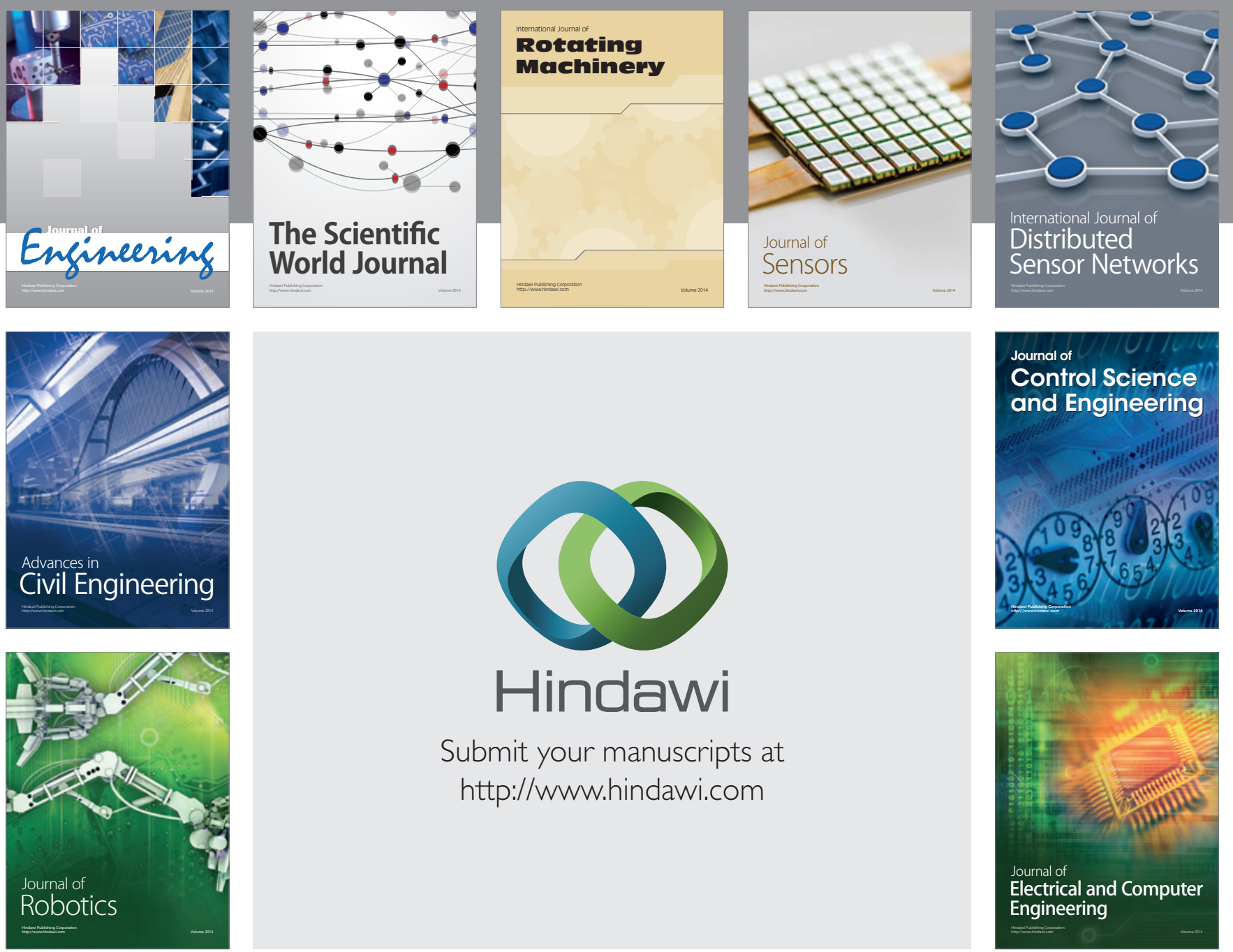

Submit your manuscripts at

http://www.hindawi.com
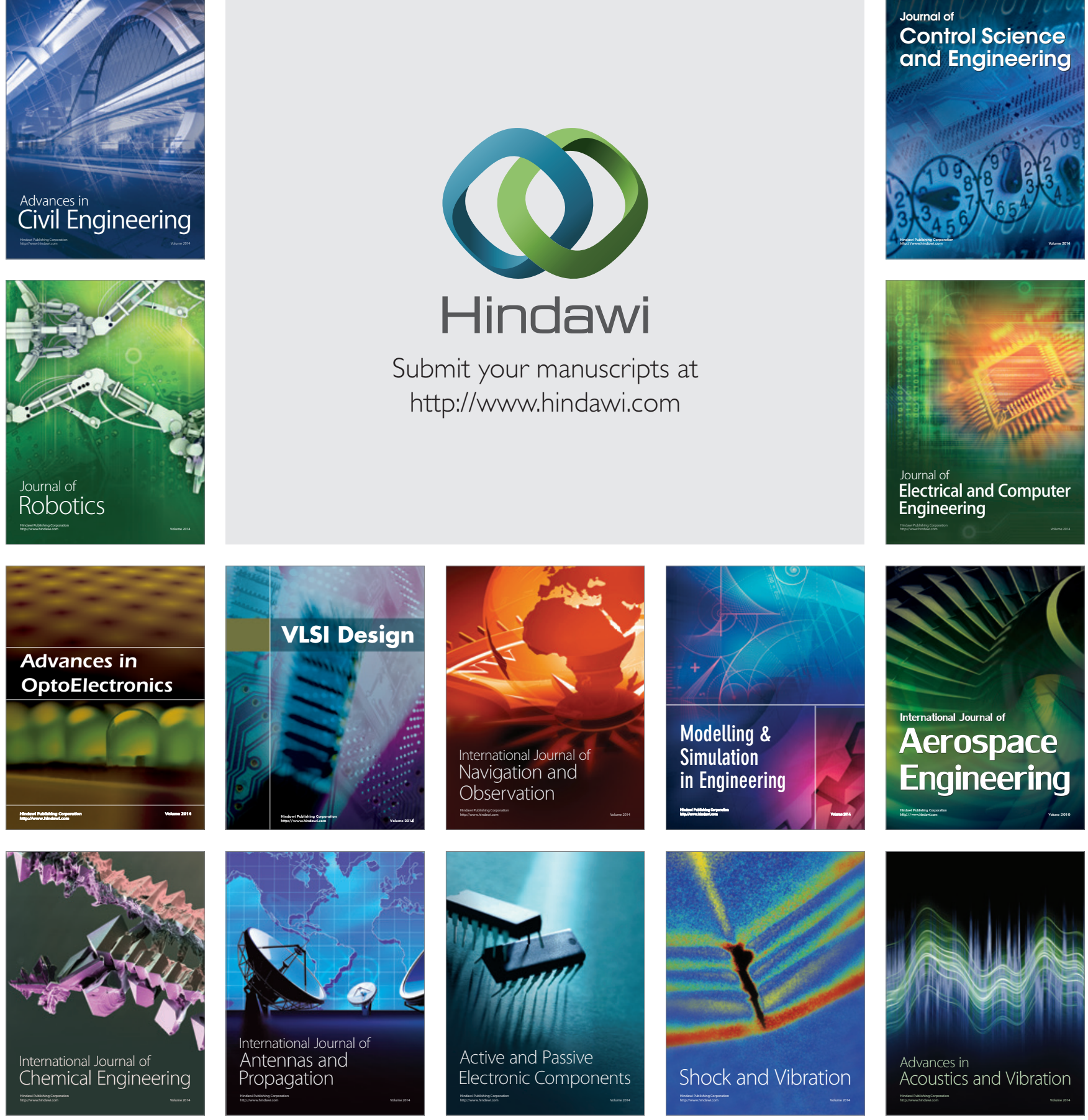